\section{Commentary on Opioid Substance Abuse among Occupational Therapy Clients}

Received: September 21, 2017; Accepted: September 27, 2017; Published: September 30, 2017

Opioid abuse has reached epidemic levels in the United States. This has been substantiated by accounts from such recognized agencies as the Centers for Disease Control and the World Health Organization, along with reports generated by individual state health agencies. While no exact figure is known as to the definite number of individuals experiencing clinically diagnosed addiction, let alone the number of those with undiagnosed dependencies, several distressing facts about this national crisis are acknowledged.

- In 2012, healthcare providers wrote over 250 million prescriptions for opioid pain medications in the United States, "enough for every American adult to have a bottle of pills" [1];

- Abuse of opioid prescription medications combined with the recent surge in use of heroin and other illicit/illegal opioid drugs has resulted in an unprecedented rise in morbidity and mortality rates, along with an upsurge in hospital and emergency department admissions [2];

- The majority of drug overdose deaths in the US now involve an opioid substance of some form [3];

- Death rates attributed to opioid substance overdose continue to rise, reaching 28,647 in 2014 [4] and 33,091 in 2015 [5];

- Yearly deaths stemming from opioid overdoses are now greater than deaths from motor vehicle accidents [6].

- This epidemic crosses all socio-economic, cultural, racial, gender boundaries; nevertheless, overdose deaths due to opioid substance abuse tend to be highest in those states with high poverty rates [7].

Health practitioners representing essentially every healthcare profession, including but not limited to physician, nursing, pharmacy, physical therapy and my own area of occupational therapy, have come to recognize the direct and ancillary impact of client opioid abuse in their clinical practice. A preliminary study of ours [8] on a small national sample of occupational therapists $(182 / 500)$ found one-third (34\%) reporting that opioid abuse is a recognized problem at their facility, an "issue of strong concern given the known abuse of opioid substances". Moreover, and rather disconcertingly, two-thirds (64\%) of those occupational therapists responding reported they had one or more clients

\section{Randy P McCombie* \\ Division of Occupational Therapy, School of Medicine, West Virginia University, USA}

\author{
*Corresponding author: \\ Randy P McCombie \\ ” jrmccombie@hsc.wvu.edu \\ PhD, OTR/L, Chair, Occupational Therapy, \\ Academic Program Director, Division of \\ Occupational Therapy, School of Medicine, \\ West Virginia University, HSC PO Box 9139, \\ Morgantown, WV 26506-9139,USA.
}

\section{Tel: 3042931954}

Citation: McCombie RP (2017) Commentary on Opioid Substance Abuse among Occupational Therapy Clients. J Drug Abuse Vol.3 No.3:22

over the past year who they "strongly suspected" of abusing opioids in some form, along with half (52\%) reporting they had one or more clients who they "knew for certain" were abusing an opioid substance. While one in five (21\%) occupational therapists also stated they personally had a client present with symptoms of overdose in the previous year, only one in twenty $(6 \%)$ indicated they were trained to respond to an overdose situation. Furthermore, the majority of these therapists reported having little or no academic or continuing education or emergency response training in regard to treating clients who present symptoms of opioid substance abuse or more distressingly, symptoms of overdose. As a result, a sizable portion of practicing occupational therapists are likely experiencing the impact and destructive effects of the nationwide opioid epidemic first hand and doing so with lack of sufficient training or understanding of effective treatment and response protocols.

Although the study in itself was limited in number of participants and overall scope it does highlight problematic issues potentially shared among all health professions. First and foremost, the opioid abuse and addiction problem is significant and in essence continuing to grow and undermine normal provision of healthcare in the US. Moreover, it is very likely that many healthcare professionals lack the education and training to respond to the crisis in their day-to-day practice. 
Consequently, health practitioners need to be informed of the extent to which the opioid abuse problem impacts their specific profession. This should be coupled with instruction in learning to recognize the signs and symptoms of opioid abuse along with training in emergency response formats for handling substance overdose. Yet, aside from individual professions promoting educational opportunities for their own practitioners, it must be recognized that dealing with the opioid problem now requires emergence of a uniquely supportive endeavor among all health care professions. Such a united venture warrants

\section{References}

1 Centers for Disease Control and Prevention (CDC) (2016) Injury prevention and control: Opioid overdose-prescription opioids.

2 Mowry JB, Spyker DA, Brooks DE, Zimmerman A, Schauben JL (2016) 2015475 annual report of the American association of poison control centers' national poison data system (NPDS): $33^{\text {rd }}$ annual report. Clin Toxicol 54: 924-1109.

3 Rudd RA, Seth P, David F, Scholl L (2016) Increases in drug and opioid-involved overdose deaths-United States, 2010-2015. MMWR Morb Mortal Wkly Rep.

4 Rudd RA, Aleshire N, Zibbell JE, Gladden RM (2016) Increases in drug and opioid overdose deaths-United States, 2000-2014. MMWR Morb Mortal Wkly Rep 64: 1378-1382. an unprecedented level of interprofessional cooperation and resolve. Addressing the problems in an isolated fashion is not sufficient to reverse the course of this epidemic. An initial step has already taken place with the Surgeon General's "Turn the Tide Campaign," essentially calling for all healthcare clinicians and academicians to raise awareness about opioid addiction [9]. It is now up to the healthcare community as a whole to formally commit itself to cooperatively addressing and ending this national crisis.

5 Rudd RA, Seth P, David F, Scholl L (2016) Increases in drug and opioid-involved overdose deaths-United States, 2010-2015. MMWR Morb Mortal Wkly Rep 65: 144501452.

6 Centers for Disease Control and Prevention (CDC) (2014) Web-based inquiry statistics query and reporting system (WISQARS).

7 Centers for Disease Control and Prevention (CDC) (2011) Vital signs: Overdoses of prescription opioid pain relievers-United States, 19992008. MMWR Morb Mortal Wkly Rep 60: 1487-1492.

8 McCombie RP, Stirling JL (2017) Opioid substance abuse among occupational therapy clients. Occup Ther Mental Health 1-12.

9 Murthy VH (n.d.) Turn the tide: The surgeon general's call to end the opioid crisis. 